\title{
VARIABILIDADE ESPACIAL E SAZONAL DA CONCENTRAÇÃO DE ELEMENTOS-TRAÇO EM SEDIMENTOS DO SISTEMA ESTUARINO DE SANTOS-CUBATÃO (SP)
}

\author{
Wanilson Luiz-Silva* \\ Instituto de Geociências, Universidade Estadual de Campinas, R. João Pandiá Calógeras, 51, 13083-970 Campinas - SP \\ Rosa Helena Ribeiro Matos e Giane Chaves Kristosch \\ Departamento de Zoologia, Universidade Estadual Paulista, 13506-900 Rio Claro - SP \\ Wilson Machado \\ Departamento de Geoquímica, Universidade Federal Fluminense, 24020-007 Niterói - RJ
}

Recebido em 22/3/05; aceito em 28/6/05; publicado na web em 20/1/06

\begin{abstract}
SPATIAL AND SEASONAL VARIABILITY OF TRACE-ELEMENT CONCENTRATIONS IN SEDIMENTS FROM THE SANTOSCUBATÃO ESTUARINE SYSTEM, SÃO PAULO, BRAZIL. Multi-element analyses of sediment samples from the Santos-Cubatão Estuarine System were carried out to investigate the spatial and seasonal variability of trace-element concentrations. The study area contains a rich mangrove ecosystem that is a habitat for tens of thousands of resident and migratory birds, some of them endangered globally. Enrichments of metals in fine-grained surface sediments are, in decreasing order, $\mathrm{Hg}, \mathrm{Mn}, \mathrm{La}, \mathrm{Ca}, \mathrm{Sr}, \mathrm{Cd}, \mathrm{Zn}$, $\mathrm{Pb}, \mathrm{Ba}, \mathrm{Cu}, \mathrm{Cr}, \mathrm{Fe}, \mathrm{Nb}, \mathrm{Y}, \mathrm{Ni}$ and $\mathrm{Ga}$, relative to pre-industrial background levels. The maximum enrichment ranged from $49(\mathrm{Hg})$ to $3.1(\mathrm{Ga})$. Mercury concentrations were greater in the Cubatão river than in other sites, while the other elements showed greater concentrations in the Morrão river. Concentrations of $\mathrm{Mn}$ were significantly greater in winter and autumn than in summer and spring. However, other elements (e.g. $\mathrm{Cd}$ and $\mathrm{Pb}$ ) showed the opposite, with greater concentrations in summer and spring. This study suggests that seasonal changes in physical and chemical conditions may affect the degree of sediment enrichment and therefore make the assessment of contamination difficult. Consequently, these processes need to be considered when assessing water quality and the potential contamination of biota.
\end{abstract}

Keywords: heavy metals; sediment geochemistry; contamination.

\section{INTRODUÇÃO}

A industrialização e a urbanização em áreas litorâneas têm contribuído para a contaminação e a degradação de ambientes estuarinos, colocando em risco o equilíbrio de importantes ecossistemas, a exemplo dos manguezais. Especialmente em função da descarga de efluentes industriais, o ambiente estuarino entre as cidades de Santos e Cubatão (estado de São Paulo) tem sido considerado um dos cenários costeiros mais seriamente contaminados do mundo ${ }^{1-5}$. Iniciado na década de 1950, o Pólo Industrial de Cubatão tornou-se o mais importante complexo petroquímico da América Latina 5 . Em 1984, a CETESB (Companhia de Tecnologia e Saneamento Ambiental), ligada à Secretaria do Meio Ambiente do governo do estado de São Paulo, deu início a um intensivo programa de controle da poluição na área desse pólo ${ }^{6}$. Desde então, alguns estudos foram realizados para identificar e determinar os níveis de contaminação no ar, na água, nos sedimentos e na biota ${ }^{1,2,6,7}$.

O papel dos sedimentos em um cenário impactado tem sido reconhecido, particularmente devido à sua capacidade de concentrar os metais-traço presentes na água ${ }^{8}$. Uma vez contaminados, os sedimentos podem representar uma fonte potencial de metais-traço para o ambiente ${ }^{9}$, mesmo após a redução ou a eliminação das emissões antrópicas. Estudos sobre a geoquímica de sedimentos no sistema estuarino de Santos-Cubatão são escassos e, embora um programa de controle da contaminação te-

*e-mail: wanilson@ige.unicamp.br nha sido colocado em prática, concentrações de metais pesados acima dos valores toleráveis por órgãos de controle ambiental têm sido documentadas nos últimos anos ${ }^{4,6}$. Luiz-Silva et al. ${ }^{4}$ mostraram condições críticas de concentração de $\mathrm{Hg}$ nos sedimentos de superfície deste estuário (em 1997 e 1998), cujos teores apresentaram-se tão elevados quanto na época das emissões de efluentes industriais sem tratamento $(1979 / 1980)^{10}$, revelando a necessidade de estudos detalhados do problema e dos riscos à biota. Adicionalmente, pouco se sabe a respeito do comportamento sazonal dos metais neste estuário, cuja concentração pode ser ampliada em determinadas épocas do ano. Por ex., estudos na área estuarina de Mai Po (Hong Kong) têm mostrado que fatores sazonais afetam a mobilidade de contaminantes metálicos na interface sedimento-água ${ }^{11}$. Particularmente, as variações temporais nas condições físico-químicas de águas intersticiais de sedimentos costeiros, como, por ex., registradas em sedimentos da Baía de Sepetiba, no Rio de Janeiro ${ }^{12,13}$, são fatores de influência reconhecidos sobre o comportamento de elementos como Fe e Mn, assim como metais-traço a eles asso$\operatorname{ciados}^{8,13}$.

O presente trabalho avaliou a variabilidade espacial e temporal das concentrações multi-elementares (incluindo-se os elementos-traço mais tóxicos) em sedimentos do sistema estuarino de Santos-Cubatão, dando continuidade a um estudo prévio realizado por Luiz-Silva et l. $^{4}$, que abordou o índice de geoacumulação de $\mathrm{Hg}$ nos sedimentos superficiais do referido sistema. Os resultados geoquímicos apresentados pretendem contribuir para a formação de base de dados para estudos futuros de monitoramento ambiental do sistema estuarino, dando suporte à aplicação de políticas públicas. 


\section{PARTE EXPERIMENTAL}

\section{Área de estudo e amostragem}

A área de estudo (Figura 1) encontra-se a leste da cidade de Cubatão e a norte da cidade de Santos, no litoral do estado de São Paulo. O setor estuarino investigado é alimentado por sedimentos drenados das encostas da Serra do Mar (localmente denominada Serra do Cubatão), a qual é geologicamente marcada por uma falha de expressão regional (falha de Cubatão) com direção NE, que põe em contato rochas de diferentes resistências à erosão, como metassedimentos que incluem xistos, filitos, quartzitos e rochas cálciosilicatadas no bloco norte e um complexo gnáissico migmatítico ao $\mathrm{sul}^{14}$. O relevo escarpado da região limita a faixa estuarina a um estreito segmento de terra, que integra a Baixada Santista.

Os rios Cubatão e Morrão são as principais vias diretas de despejos de efluentes industriais. Despejos domésticos, lixões e aterros sanitários têm afetado mais diretamente os rios Cubatão, Cascalho e Casqueiro. O rio Cubatão, em particular, tem sido afetado por despejos de indústrias químicas, petroquímicas e de fertilizantes, enquanto o rio Morrão tem sofrido, especialmente, o impacto de efluentes de uma grande siderúrgica ${ }^{6}$. As fontes potenciais de contaminação e a natureza dos compostos lançados no sistema estuarino investigado estão detalhadas em relatório da CETESB ${ }^{6}$.

Dominado por um clima tropical a subtropical ${ }^{3}$, o sistema estuarino de Santos-Cubatão possui um exuberante manguezal, que constitui um valioso fornecedor de alimentos (peixes e crustáceos) para consumo humano e serve de abrigo, área de forrageamento e reprodução para dezenas de milhares de aves residentes e migratórias do hemisfério norte ${ }^{15,16}$. Os sítios de amostragem considerados aqui também foram monitorados durante o mesmo período (19971998) com objetivos ornitológicos ${ }^{14,15}$, demonstrando-se o valor ecológico da área estudada, que abriga espécies ameaçadas de extinção em escala global, como o Guará (Eudocimus ruber $)^{15}$. Além de causar a deterioração da qualidade ambiental local, a contaminação neste estuário pode afetar a Baía de Santos adjacente, que abriga importante porto e constitui uma zona de particular interesse econômico (turismo e pesca).

Sedimentos superficiais foram coletados em 15 sítios de amostragem (Figura 1), distribuídos no Largo do Caneu (sítios GD, GE e GF) e em alguns de seus tributários, como os rios Cascalho (sítios GG, GH e GI), Casqueiro (sítios RA, RB e RC), Cubatão (sítios GA, GB e GC), Capivari (sítio GL), Morrão (sítio GJ) e Pedreira (sítio GK). Com o objetivo de representar e avaliar a variabilidade temporal e espacial nas concentrações dos elementostraço, as coletas foram realizadas em diversas épocas do ano. Os sedimentos dos rios Capivari, Morrão e Pedreira foram amostrados nos meses de março, junho, setembro e dezembro de 1998, enquanto os demais rios foram amostrados mensalmente, no período de setembro de 1997 a agosto de 1998.

Cada ponto de coleta foi representado por uma área de $\sim 20$ $\mathrm{m}^{2}$, dentro da qual amostras compostas de sedimentos superficiais $(\sim 2-3 \mathrm{~cm})$ foram coletadas manualmente, com uso de luvas de borracha, durante a maré baixa.

No rio Casqueiro, sítio de amostragem RA (Figura 1), também foi coletado (em junho de 2001) um testemunho de sedimento com $31 \mathrm{~cm}$ de profundidade (testemunho CAS-1), utilizando-se tubo de PVC (10 cm de diâmetro). O testemunho foi amostrado em intervalos de $2 \mathrm{~cm}$ até a profundidade de $12 \mathrm{~cm}$ e, a partir de então, em intervalos de 3 a $6 \mathrm{~cm}$. Os sedimentos em contato com as paredes do tubo não foram utilizados para as análises. As amostras deste testemunho representaram importante base para identificar valores de concentração multi-elementar correspondentes a períodos anteriores ao início da industrialização da área de estudo ("background" litogênico), procedimento este já realizado em outras áreas da costa sudeste brasileira ${ }^{17-19}$.

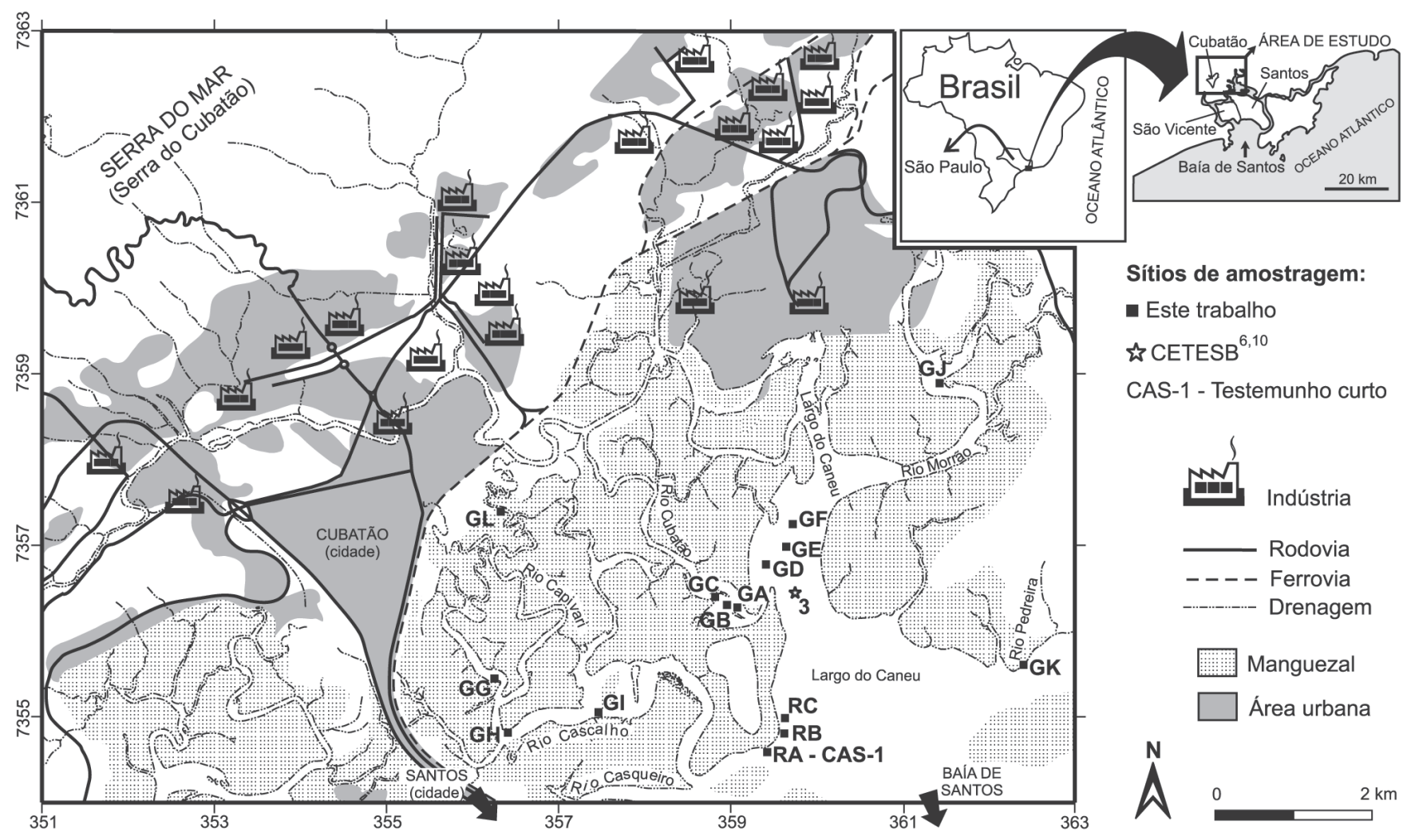

Figura 1. Mapa simplificado do sistema estuarino de Santos-Cubatão com a localização dos sítios de amostragem. Adaptado da ref. 4 


\section{Tratamento das amostras e métodos analíticos}

Em laboratório, amostras dos sedimentos foram secas (a 30 ${ }^{\circ} \mathrm{C}$ ), homogeneizadas, quarteadas e a fração $<63 \mu \mathrm{m}$ (silte-argila) foi separada, utilizando-se peneiras de nylon. Essa fração foi utilizada nas análises químicas, buscando-se evitar o efeito diluidor das frações granulométricas mais grossas dos sedimentos sobre sua composição química ${ }^{20,21}$. Um peneiramento úmido foi também realizado ( $10 \mathrm{~g}$ de amostra) apenas para melhor quantificar a fração $<63 \mu \mathrm{m}^{20}$. Análises químicas multi-elementares (Ag, Al, As, Ba, Bi, Ca, Cd, Co, Cr, Cu, Fe, Ga, Hg, K, La, Li, Mg, Mn, Nb, $\mathrm{Ni}, \mathrm{Pb}, \mathrm{Sb}, \mathrm{Sc}, \mathrm{Sr}, \mathrm{Ta}, \mathrm{Te}, \mathrm{V}, \mathrm{Y}$ e Zn) foram realizadas no Laboratório ALS Chemex (Vancouver, Canadá), após digestão de 0,5 g de sedimento em água régia, à temperatura de $90^{\circ} \mathrm{C}$, durante $2,5 \mathrm{~h}$. A concentração do $\mathrm{Hg}$ foi determinada via AAS com geração de vapor a frio, enquanto as concentrações dos demais elementos foram determinadas via ICPAES. Alguns elementos, com concentração muito baixa, foram analisados via ICPMS (Ag, As, Bi, Cd, Ga, Sb, Sc, Ta e Te), particularmente nas amostras do testemunho CAS-1. Brancos analíticos, 2 materiais de referência certificados (CANMET STSD-4 e CANMET LKSD-3), 5 amostras de referência do Laboratório ALS Chemex e 13 duplicatas das amostras da área de estudo foram analisados para controle da qualidade analítica. As análises dos materiais de referência apresentaram diferenças percentuais relativas (DPR) inferiores a 15\%, com exceção do Cd (DPR entre 6 e 50\%) e La (DPR entre 7 e 37\%) nas análises por ICPAES, cujas concentrações nas amostras de referência eram muito baixas. Análises das amostras duplicatas apresentaram DPR aceitáveis (inferiores a $15 \%$ ), considerando que diferenças de até $30 \%$ são consideradas satisfatórias para controle de duplicatas analíticas ${ }^{22}$.

\section{Tratamento dos dados}

O "background" geoquímico natural (litogênico) para a área investigada foi calculado a partir dos valores médios das concentrações dos elementos nos sedimentos das camadas inferiores do testemunho CAS-1. Este método tem sido bastante aplicado ${ }^{23,24}$ até mesmo para estimativa de valores de "background" para escalas regionais $^{25}$.

As anomalias geoquímicas nos sedimentos de superfície foram avaliadas por meio de coeficientes de variação, frequiências cumulativas relativas e fatores de enriquecimento. Nos cálculos dos coeficientes de variação e nos gráficos de frequiências cumulativas relativas, os dados geoquímicos do testemunho CAS-1 foram incluídos, totalizando 166 amostras avaliadas na área de estudo. O coeficiente de variação ( $\mathrm{CV}$ = desvio padrão/média) é capaz de fornecer uma boa idéia acerca da distribuição dos dados geoquímicos ${ }^{26}$. Valores de CV menores que 0,3 indicam distribuição normal ou aproximadamente normal e sugerem ausência de anomalias (teores mais homogêneos). Para valores de CV especialmente maiores que 0,8 , a distribuição fortemente assimétrica (heterogeneidade nos dados) sugere anomalias geoquímicas mais significativas $^{26}$. Por outro lado, uma faixa de valores de CV entre 0,4 e 0,7 não permite qualquer conclusão ${ }^{27}$.

Freqüências cumulativas relativas ou somas cumulativas, de acordo com o método gráfico proposto por Lepeltier ${ }^{28}$ e levemente modificado por Matschullat et al. ${ }^{29}$, baseiam-se no pressuposto de que concentrações de elementos-traço possuem uma distribuição log-normal. Projetando-se as somas cumulativas vs. concentração, em escala bi-logarítmica, um desvio da distribuição log-normal pode ser percebido como uma inflexão na parte superior da curva, representando uma anomalia. Este procedimento foi desenvolvido para prospecção geoquímica de bens minerais, mas pode ser apli- cado para determinar anomalias geoquímicas em sedimentos impactados ${ }^{29}$.

Uma vez definidas as heterogeneidades geoquímicas com base nos critérios acima, a intensidade das anomalias foi expressa por meio do fator de enriquecimento (FE):

$\mathrm{FE}=\left(\mathrm{C}_{\mathrm{i}} / \mathrm{C}_{\mathrm{R}}\right)_{\text {amostra }} /\left(\mathrm{C}_{\mathrm{i}} / \mathrm{C}_{\mathrm{R}}\right)_{\text {"background" }}$

Este fator, baseado em Gresens ${ }^{30}$, quantifica a concentração do elemento de interesse $\left(\mathrm{C}_{\mathrm{i}}\right)$ na amostra, em relação ao "background" geoquímico, cujas heterogeneidades litogênicas (efeito das variações granulométricas e mineralógicas) são minimizadas com a introdução na equação do elemento normalizador $\left(C_{R}=\right.$ concentração do elemento de referência). Dentre os normalizadores comumente empregados, o Li foi adotado neste trabalho por apresentar distribuição uniforme nas amostras analisadas e já ter sido testado em outros estudos ${ }^{31}$.

Nos rios Cascalho, Casqueiro, Cubatão e no Largo do Caneu, a variabilidade geoquímica sazonal dos elementos foi avaliada em cinco estações (do inverno/1997 ao inverno/1998), enquanto nos rios Capivari, Morrão e Pedreira quatro estações foram consideradas (do verão/1998 à primavera/1998).

\section{RESULTADOS E DISCUSSÃO}

\section{Granulometria}

A proporção da fração silte-argila $(<63 \mu \mathrm{m})$ dos sedimentos (peneiramento úmido) é mostrada na Tabela 1. A porcentagem desta fração variou bastante entre os sítios de amostragem (variação espacial) e, em vários casos, variou também dentro de cada sítio. Os sedimentos silto-argilosos predominaram, durante todo o período de amostragem, nos rios Morrão e Pedreira (a fração $<63 \mu \mathrm{m}$ variou de 86 a 89\%). Nas demais drenagens investigadas, a variabilidade no conteúdo da fração $<63 \mu \mathrm{m}$, geralmente subordinado ao da fração arenosa, foi expressiva, oscilando entre 2 e 4 fatores. Estas variações na granulometria de um mesmo sítio (variações temporais) sugerem que os sedimentos estudados tiveram sua composição alterada pelo transporte das águas costeiras, entre os períodos de amostragem.

\section{“Background" e anomalias geoquímicas}

A Tabela 1 apresenta as médias, os desvios-padrão e os intervalos de concentração de $\mathrm{Al}, \mathrm{As}, \mathrm{Ba}, \mathrm{Ca}, \mathrm{Cd}, \mathrm{Co}, \mathrm{Cr}, \mathrm{Cu}, \mathrm{Fe}, \mathrm{Ga}$, $\mathrm{Hg}, \mathrm{K}, \mathrm{La}, \mathrm{Li}, \mathrm{Mg}, \mathrm{Mn}, \mathrm{Nb}, \mathrm{Ni}, \mathrm{Pb}, \mathrm{Sc}, \mathrm{Sr}, \mathrm{V}$, Y e Zn dos sedimentos de superfície das drenagens investigadas. Elementos não mostrados como Ag (limite de detecção $\left.=0,2 \mathrm{mg} \mathrm{kg}^{-1}\right), \mathrm{Bi}\left(5 \mathrm{mg} \mathrm{kg}^{-1}\right)$, $\mathrm{Sb}\left(5 \mathrm{mg} \mathrm{kg}^{-1}\right)$, Ta $\left(10 \mathrm{mg} \mathrm{kg}^{-1}\right)$ e Te $\left(10 \mathrm{mg} \mathrm{kg}^{-1}\right)$ apresentaram respostas analíticas abaixo do limite instrumental (ICPAES). Devido ao número significativo de análises com valores abaixo desta limitação, as concentrações médias de $\mathrm{Cd}$ (limite de detecção = $\left.0,2 \mathrm{mg} \mathrm{kg}^{-1}\right)$ e Sc $\left(5 \mathrm{mg} \mathrm{kg}^{-1}\right)$ não foram calculadas nos sítios de amostragem, com exceção dos rios Morrão (Cd) e Pedreira (Sc). Em algumas amostras, valores abaixo do limite de detecção para As (três valores $<0,2 \mathrm{mg} \mathrm{kg}^{-1}$ ) e $\mathrm{Ga}$ (sete valores $<0,2 \mathrm{mg} \mathrm{kg}^{-1}$ ) também foram encontrados, os quais foram descartados no cálculo das médias. Este procedimento não afetou significativamente a média destes elementos, tendo em vista que os outros valores de concentração eram baixos e próximos ao limite de detecção.

A Tabela 2 apresenta os dados da geoquímica do testemunho CAS-1. Valores médios referentes ao "background" geoquímico litogênico foram calculados em sedimentos abaixo dos $12 \mathrm{~cm}$ de 
Tabela 1. Dados geoquímicos da fração < $63 \mu \mathrm{m}$ de sedimentos de superfície da área de estudo (amostragem 1997-1998; análises por ICPAES). $\mathrm{n}$ = número de amostras, $\mathrm{DP}=$ desvio-padrão e $\mathrm{ND}=$ não determinada (valores inferiores ao limite analítico)

\begin{tabular}{|c|c|c|c|c|c|c|c|c|c|c|c|c|c|c|}
\hline & \multirow{2}{*}{\multicolumn{2}{|c|}{$\begin{array}{l}\text { Rio Casqueiro }(\mathrm{n}=36 \\
\text { Média } \pm \text { DP Intervalo }\end{array}$}} & \multicolumn{2}{|c|}{ 5) Rio Cubatão $(n=36)$} & \multicolumn{2}{|c|}{ Largo do Caneu $(\mathrm{n}=36)$} & \multicolumn{2}{|c|}{ Rio Cascalho $(\mathrm{n}=36)$} & \multicolumn{2}{|c|}{ Rio Capivari $(\mathrm{n}=4)$} & \multicolumn{2}{|c|}{ Rio Pedreira $(n=4)$} & \multicolumn{2}{|c|}{ Rio Morrão $(n=4)$} \\
\hline & & & Média \pm DP & Intervalo & Média \pm DP & Intervalo & Média \pm DF & P Intervalo & Média \pm DP & Intervalo & Média \pm DP & Intervalo & Média \pm DP & Intervalo \\
\hline $\mathrm{Al}(\%)$ & $2,6 \pm 0,5$ & $1,3-3,4$ & $3,2 \pm 0,4$ & $2,3-3,9$ & $3,1 \pm 0,5$ & $1,6-4,1$ & $2,9 \pm 0,3$ & $2,2-3,3$ & $2,9 \pm 0,4$ & $2,5-3,3$ & $3,3 \pm 0,1$ & $3,1-3,4$ & $2,5 \pm 0,7$ & $1,8-3,1$ \\
\hline As & $7,6 \pm 1,2$ & $<5-10$ & $7,7 \pm 1,6$ & $5-12$ & $7,1 \pm 1$ & $<5-9$ & $12 \pm 1,8$ & $8-15$ & $9,5 \pm 1,3$ & $8-11$ & $7,3 \pm 1,3$ & $6-9$ & $8 \pm 3,6$ & $5-13$ \\
\hline $\mathrm{Ba}$ & $42 \pm 15$ & $17-104$ & $97 \pm 27$ & $38-184$ & $92 \pm 17$ & $41-128$ & $57 \pm 7$ & $40-69$ & $58 \pm 2$ & $57-60$ & $35 \pm 2$ & $33-38$ & $186 \pm 14$ & $166-196$ \\
\hline $\mathrm{Ca}(\%)$ & $0,26 \pm 0,06$ & $0,16-0,45$ & $0,21 \pm 0,05$ & $0,09-0,35$ & $0,28 \pm 0,06$ & $0,06-0,39$ & $0,25 \pm 0,05$ & $0,11-0,33$ & $0,25 \pm 0,04$ & $0,2-0,29$ & $0,35 \pm 0,07$ & $0,26-0,43$ & $3,12 \pm 1,05$ & $2,24-4,37$ \\
\hline $\mathrm{Cd}$ & ND & $<0,2-0,3$ & ND & $<0,2-0,3$ & ND & $<0,2-0,3$ & ND & $<0,2-0,3$ & $<0,2$ & $<0,2$ & $<0,2$ & $<0,2$ & $0,73 \pm 0,61$ & $0,2-1,6$ \\
\hline Co & $7,2 \pm 1,4$ & $4-11$ & $10,3 \pm 1,3$ & $7-13$ & $9,4 \pm 0,9$ & $7-11$ & $10 \pm 1$ & $8-13$ & $10 \pm 1,3$ & $9-12$ & $7,3 \pm 0,5$ & $7-8$ & $13 \pm 1$ & $12-14$ \\
\hline $\mathrm{Cr}$ & $38 \pm 7$ & $23-51$ & $45 \pm 5$ & $30-55$ & $44 \pm 6$ & $25-56$ & $46 \pm 5$ & $37-66$ & $40 \pm 3$ & $36-44$ & $42 \pm 1$ & $40-43$ & $127 \pm 10$ & $117-138$ \\
\hline $\mathrm{Cu}$ & $31 \pm 11$ & $18-73$ & $32 \pm 7$ & $18-57$ & $31 \pm 7$ & $13-58$ & $38 \pm 11$ & $21-89$ & $31 \pm 5$ & $25-38$ & $28 \pm 8$ & $22-39$ & $91 \pm 21$ & $68-109$ \\
\hline $\mathrm{Fe}(\%)$ & $3,3 \pm 0,5$ & $1,9-4,2$ & $3,9 \pm 0,5$ & $2,7-5,2$ & $4,3 \pm 0,6$ & $2,1-5,4$ & $4,9 \pm 0,8$ & $3,3-7,1$ & $3,8 \pm 0,6$ & $3-4,3$ & $3,6 \pm 0,04$ & $3,6-3,7$ & $>10$ & $>10$ \\
\hline $\mathrm{Ga}$ & $3,5 \pm 0,7$ & $<2-5$ & $4,3 \pm 0,9$ & $2-6$ & $3,9 \pm 0,9$ & $2-6$ & $3,5 \pm 0,7$ & $2-5$ & $3,3 \pm 0,6$ & $<2-4$ & $5,3 \pm 0,5$ & $5-6$ & $11,8 \pm 3,5$ & $8-16$ \\
\hline $\mathrm{Hg}$ & $1,04 \pm 0,6$ & $0,25-3,04$ & $1,73 \pm 1,57$ & $0,54-6,77$ & $1,04 \pm 0,26$ & $0,47-1,79$ & $0,39 \pm 0,11$ & $0,1-0,57$ & $0,31 \pm 0,11$ & $0,18-0,4$ & $0,59 \pm 0,02$ & $0,57-0,61$ & $0,45 \pm 0,09$ & $0,37-0,58$ \\
\hline $\mathrm{K}(\%)$ & $0,4 \pm 0,1$ & $0,3-0,6$ & $0,5 \pm 0,1$ & $0,4-0,6$ & $0,5 \pm 0,1$ & $0,3-0,6$ & $0,5 \pm 0,1$ & $0,4-0,6$ & $0,5 \pm 0,1$ & $0,5-0,6$ & 0,5 & 0,5 & $0,2 \pm 0,1$ & $0,2-0,3$ \\
\hline $\mathrm{La}$ & $23 \pm 5,8$ & $12-43$ & $34 \pm 8,8$ & $17-49$ & $36 \pm 7$ & $11-46$ & $26 \pm 3,6$ & $16-32$ & $25 \pm 1,4$ & $23-26$ & $24 \pm 0,5$ & $24-25$ & $214 \pm 177$ & $75-450$ \\
\hline $\mathrm{Li}$ & $31 \pm 6$ & $15-42$ & $38 \pm 5$ & $25-47$ & $38 \pm 5$ & $21-49$ & $37 \pm 5$ & $27-52$ & $47 \pm 9$ & $34-56$ & $37 \pm 1$ & $36-37$ & $21 \pm 5$ & $16-25$ \\
\hline $\operatorname{Mg}(\%)$ & b) $0,7 \pm 0,1$ & $0,5-0,9$ & $0,7 \pm 0,1$ & $0,5-0,9$ & $0,8 \pm 01$ & $0,4-0,9$ & $0,8 \pm 0,1$ & $0,6-1$ & $0,9 \pm 0,1$ & $0,8-0,9$ & $0,9 \pm 0,1$ & $0,8-0,9$ & $0,8 \pm 0,1$ & $0,8-0,9$ \\
\hline Mn & $236 \pm 39$ & $160-332$ & $276 \pm 43$ & 206-396 & $320 \pm 44$ & $221-421$ & $411 \pm 172$ & $241-1045$ & $518 \pm 284$ & $302-935$ & $238 \pm 7$ & $228-243$ & $4526 \pm 1291$ & $3276-5699$ \\
\hline $\mathrm{Nb}$ & $3,1 \pm 0,8$ & $1-5$ & $4 \pm 0,8$ & $2-5$ & $3,6 \pm 0,7$ & $2-5$ & $4,3 \pm 0,7$ & $3-5$ & $4,3 \pm 0,5$ & $4-5$ & $3,8 \pm 0,5$ & $3-4$ & $8 \pm 2,9$ & $5-11$ \\
\hline $\mathrm{Ni}$ & $17 \pm 3,5$ & $10-24$ & $22 \pm 2,3$ & $15-27$ & $21 \pm 2,6$ & $12-25$ & $23 \pm 2,7$ & $18-32$ & $21 \pm 2,2$ & $19-24$ & $17 \pm 0,5$ & $17-18$ & $36 \pm 2,9$ & $33-39$ \\
\hline $\mathrm{Pb}$ & $20 \pm 5$ & $9-29$ & $22 \pm 4$ & $14-28$ & $27 \pm 7$ & $9-46$ & $23 \pm 5$ & $14-32$ & $18 \pm 4$ & $13-21$ & $25 \pm 2$ & $22-27$ & $104 \pm 16$ & $92-127$ \\
\hline $\mathrm{Sc}$ & ND & $<5-6$ & ND & $<5-8$ & ND & $<5-6$ & ND & $<5-6$ & ND & $<5-6$ & $5,3 \pm 0,5$ & $5-6$ & ND & $<5-11$ \\
\hline $\mathrm{Sr}$ & $41 \pm 7$ & $30-55$ & $40 \pm 10$ & $18-51$ & $52 \pm 11$ & $13-70$ & $47 \pm 7$ & $25-57$ & $44 \pm 11$ & $30-53$ & $58 \pm 4$ & $53-62$ & $445 \pm 276$ & 214-764 \\
\hline V & $33 \pm 6$ & $20-45$ & $42 \pm 5$ & $31-58$ & $39 \pm 4$ & $24-46$ & $48 \pm 4$ & $37-56$ & $43 \pm 5$ & $39-50$ & $37 \pm 2$ & $35-39$ & $50 \pm 4$ & $47-55$ \\
\hline Y & $10 \pm 2,6$ & $6-13$ & $12 \pm 1,4$ & $9-15$ & $12 \pm 1,7$ & $6-15$ & $11 \pm 0,8$ & $8-12$ & $11 \pm 0,6$ & $10-11$ & $10 \pm 0,5$ & $10-11$ & $28 \pm 17$ & $14-48$ \\
\hline $\mathrm{Zn}$ & $86 \pm 14$ & 53-109 & $94 \pm 12$ & $56-118$ & $110 \pm 18$ & $54-148$ & $112 \pm 18$ & $74-146$ & $80 \pm 12$ & $66-94$ & $93 \pm 5,6$ & 88-99 & $440 \pm 31$ & $406-476$ \\
\hline \multicolumn{15}{|c|}{$<63 \mu \mathrm{m}(\%)$} \\
\hline & $51 \pm 10$ & $26-76$ & $62 \pm 16$ & 24-79 & $49 \pm 14$ & $14-51$ & $42 \pm 18$ & $20-82$ & $53 \pm 12$ & $35-60$ & $88 \pm 1$ & $87-89$ & $87 \pm 1$ & $86-88$ \\
\hline
\end{tabular}

As concentrações estão em $\mathrm{mg} \mathrm{kg}^{-1}$ quando não especificadas. O conteúdo da fração $<63 \mathrm{~mm}$ nos sedimentos está apresentado no final da tabela. Incerteza de até $15 \%$ é admitida para os dados.

profundidade, a partir da qual as concentrações foram, geralmente, mais homogêneas e contrastantes com os teores mais elevados no topo $^{23,24}$. A taxa de sedimentação para o estuário investigado, estimada por Hoshika et al. ${ }^{32}$, sendo da ordem de $1,7 \mathrm{~mm}^{2} \mathrm{no}^{-1}$, sugere que, neste local, abaixo dessa profundidade os sedimentos correspondem a períodos pré-industriais. Esses valores de "background" são semelhantes ou inferiores à média global dos folhelhos ${ }^{33}$ e estão sendo encontrados, também, em outro setor deste estuário (estudo em andamento). Sedimentos mais profundos (120$150 \mathrm{~cm}$ ) no rio Morrão (dados inéditos) estão mostrando geoquímica multi-elementar muito parecida com aquela do testemunho CAS-1 (Tabela 2); por ex. (média de três amostras): Ba $\left(50 \pm 1 \mathrm{mg} \mathrm{kg}^{-1}\right)$, $\mathrm{Ca}(0,18 \pm 0,03 \%), \mathrm{Cd}\left(0,1 \pm 0,03 \mathrm{mg} \mathrm{kg}^{-1}\right), \mathrm{Cr}\left(39 \pm 2 \mathrm{mg} \mathrm{kg}^{-1}\right), \mathrm{Cu}$ $\left(15 \pm 2 \mathrm{mg} \mathrm{kg}^{-1}\right), \mathrm{Fe}(3,1 \pm 0,2 \%), \mathrm{Ga}\left(10 \pm 0,7 \mathrm{mg} \mathrm{kg}^{-1}\right), \mathrm{Hg}(0,13$ $\left.\pm 0,1 \mathrm{mg} \mathrm{kg}^{-1}\right), \mathrm{La}\left(30 \pm 5 \mathrm{mg} \mathrm{kg}^{-1}\right), \mathrm{Mn}\left(208 \pm 6 \mathrm{mg} \mathrm{kg}^{-1}\right), \mathrm{Nb}(3,33$ $\left.\pm 0,23 \mathrm{mg} \mathrm{kg}^{-1}\right), \mathrm{Ni}\left(19 \pm 2 \mathrm{mg} \mathrm{kg}^{-1}\right), \mathrm{Pb}\left(20 \pm 0,6 \mathrm{mg} \mathrm{kg}^{-1}\right), \mathrm{Sr}(58$ $\left.\pm 5 \mathrm{mg} \mathrm{kg}^{-1}\right)$, Y $\left(16 \pm 2 \mathrm{mg} \mathrm{kg}^{-1}\right)$ e Zn $\left(69 \pm 4 \mathrm{mg} \mathrm{kg}^{-1}\right)$. Estes dados foram obtidos via ICPAES e ICPMS, utilizando-se o mesmo tratamento aplicado às amostras deste trabalho.

Analisando o mapa geológico ${ }^{34}$ e a carta topográfica ${ }^{35}$ da região de Cubatão, nota-se que a similaridade entre o "background" geoquímico de sedimentos de rios espacialmente tão distintos (rios Casqueiro e Morrão) pode ser atribuída à distribuição dos corpos litológicos fontes destes materiais e à configuração do sistema estuarino investigado. Os corpos litológicos encontram-se alongados na direção NE na Serra do Cubatão e são erodidos por drenagens ortogonais a esse alinhamento nas escarpas. Estas drenagens alimentam um conjunto de rios paralelos à direção da serra, o qual evolui para padrão anastomosado à medida que adentra na parte baixa do estuário, onde a quebra de relevo propicia maior deposição que erosão. O conjunto complexo de canais divergentes e convergentes, separados por ilhas ou elevações, deve contribuir para a mistura de sedimentos, possibilitando um "background" geoquímico litogênico mais uniforme para a área.

As concentrações de Ag, Bi, Sb, Ta e Te, inferiores aos limites de detecção (ICPAES) nas amostras de sedimentos de superfície, mostraram-se baixas e uniformes ao longo do testemunho CAS-1 (ICPMS - Tabela 2), sugerindo que estes elementos não representam casos de anomalia, pelo menos no domínio do rio Casqueiro.

Os elementos representados na Tabela 1 podem ser agrupados em duas categorias, baseadas nos coeficientes de variação e nas frequiências cumulativas relativas: aqueles com teores estatisticamente anômalos (distribuição assimétrica) e aqueles cujos teores são mais homogêneos (distribuição aproximadamente normal/lognormal). No segundo caso, foram considerados o Al (média geral incluindo o testemunho CAS-1 $=2,9 \pm 0,5 \%)$, As $(8,6 \pm 2,8 \mathrm{mg}$ $\left.\mathrm{kg}^{-1}\right), \mathrm{Co}\left(9,3 \pm 1,8 \mathrm{mg} \mathrm{kg}^{-1}\right), \mathrm{K}(0,5 \pm 0,1 \%), \mathrm{Li}\left(36 \pm 7 \mathrm{mg} \mathrm{kg}^{-1}\right)$, $\mathrm{Mg}(0,8 \pm 0,1 \%), \mathrm{Sc}\left(4,2 \pm 1,7 \mathrm{mg} \mathrm{kg}^{-1}\right)$ e V $\left(41 \pm 7 \mathrm{mg} \mathrm{kg}^{-1}\right)$. Estes elementos apresentaram baixos coeficientes de variação (entre 0,1 e 0,3 ; com exceção do Sc) e suas concentrações devem refletir as litologias fontes dos sedimentos. Esta avaliação é consistente com a similaridade entre os níveis de concentração mostrados na Tabela 1 e aqueles encontrados ao longo do perfil do testemunho CAS1 (Tabela 2). Exemplos gráficos de distribuição (aproximadamente) log-normal, utilizando Al, K, Li e V, são mostrados na Figura 2.

Os fatores de enriquecimento (FE) dos elementos geoquimicamente não anômalos ( $\mathrm{Li}=$ elemento de referência) foram inferiores a 3,0. Assim, considerando que até três fatores de enrique- 
Tabela 2. Dados geoquímicos (fração $<63 \mu \mathrm{m}$ ) de sedimentos do testemunho CAS-1 (rio Casqueiro) coletados em 2001

\begin{tabular}{|c|c|c|c|c|c|c|c|c|c|c|}
\hline & & & & & ndidad & & & & & \\
\hline & & & & & & & "Ba & ound" ge & dímico & \\
\hline & $0-2$ & $2-4$ & $4-6$ & $6-8$ & 8-10 & $10-12$ & $12-15$ & $15-21$ & $21-25$ & $25-31$ \\
\hline$\overline{\mathrm{Ag}}$ & 0,1 & 0,09 & 0,08 & 0,05 & 0,04 & 0,04 & 0,04 & 0,04 & 0,04 & 0,04 \\
\hline $\mathrm{Al}(\%)$ & 1,8 & 1,8 & 2 & 2,2 & 2,2 & 2,2 & 2,1 & 2,2 & 2,1 & 2,1 \\
\hline As & 10 & 11 & 14 & 16 & 15 & 17 & 14 & 14 & 13 & 12 \\
\hline $\mathrm{Ba}$ & 40 & 30 & 30 & 30 & 30 & 30 & 30 & 30 & 30 & 30 \\
\hline $\mathrm{Bi}$ & 0,2 & 0,21 & 0,23 & 0,19 & 0,2 & 0,21 & 0,19 & 0,19 & 0,18 & 0,18 \\
\hline $\mathrm{Ca}(\%)$ & 0,5 & 0,44 & 0,34 & 0,3 & 0,34 & 0,35 & 0,3 & 0,32 & 0,22 & 0,25 \\
\hline $\mathrm{Cd}$ & 0,2 & 0,19 & 0,17 & 0,14 & 0,12 & 0,11 & 0,12 & 0,11 & 0,11 & 0,11 \\
\hline $\mathrm{Co}$ & 7,8 & 7,6 & 8 & 8 & 8,2 & 8,2 & 7,5 & 7,6 & 8 & 7,3 \\
\hline $\mathrm{Cr}$ & 32 & 32 & 33 & 32 & 33 & 32 & 31 & 31 & 31 & 31 \\
\hline $\mathrm{Cu}$ & 21 & 21 & 20 & 16 & 16 & 15 & 14 & 14 & 13 & 12 \\
\hline $\mathrm{Fe}(\%)$ & 3 & 2,9 & 3,2 & 3,2 & 3 & 3,1 & 3 & 2,9 & 2,9 & 2,7 \\
\hline $\mathrm{Ga}$ & 6 & 5,8 & 6,4 & 6,7 & 6,9 & 6,9 & 6,3 & 6,4 & 6,5 & 6,1 \\
\hline $\mathrm{Hg}$ & 0,5 & 0,53 & 0,46 & 0,94 & 0,21 & 0,14 & 0,17 & 0,15 & 0,09 & 0,08 \\
\hline $\mathrm{K}(\%)$ & 0,4 & 0,4 & 0,4 & 0,5 & 0,5 & 0,5 & 0,5 & 0,5 & 0,5 & 0,5 \\
\hline $\mathrm{La}$ & 23 & 22 & 22 & 21 & 21 & 21 & 19 & 19 & 21 & 19 \\
\hline $\mathrm{Li}$ & 27 & 26 & 30 & 33 & 34 & 35 & 30 & 33 & 35 & 32 \\
\hline $\operatorname{Mg}(\%)$ & 0,7 & 0,7 & 0,8 & 0,9 & 0,9 & 0,9 & 0,9 & 0,9 & 0,9 & 0,8 \\
\hline $\mathrm{Mn}$ & 214 & 220 & 239 & 227 & 230 & 232 & 238 & 231 & 220 & 213 \\
\hline $\mathrm{Nb}$ & 2,2 & 2,1 & 2,2 & 2 & 2 & 1,9 & 1,8 & 2,2 & 2,1 & 2,1 \\
\hline $\mathrm{Ni}$ & 16 & 24 & 16 & 14 & 15 & 14 & 13 & 15 & 16 & 15 \\
\hline $\mathrm{Pb}$ & 30 & 29 & 19 & 15 & 16 & 14 & 14 & 14 & 14 & 15 \\
\hline $\mathrm{Sb}$ & 0,4 & 0,46 & 0,48 & 0,41 & 0,38 & 0,37 & 0,33 & 0,3 & 0,21 & 0,2 \\
\hline $\mathrm{Sc}$ & 4,6 & 4,5 & 4,9 & 5,4 & 5,5 & 5,7 & 5,2 & 6,3 & 6,3 & 5,9 \\
\hline $\mathrm{Sr}$ & 60 & 59 & 60 & 59 & 60 & 62 & 57 & 57 & 43 & 43 \\
\hline $\mathrm{Ta}$ & $<0,01$ & $<0,01$ & $<0,01$ & $<0,01$ & 0,01 & 0,01 & 0,01 & 0,01 & $<0,01$ & $<0,01$ \\
\hline $\mathrm{Te}$ & 0,1 & 0,07 & 0,07 & 0,06 & 0,06 & 0,06 & 0,06 & 0,05 & 0,04 & 0,04 \\
\hline $\mathrm{V}$ & 32 & 32 & 37 & 40 & 41 & 42 & 40 & 40 & 35 & 36 \\
\hline $\mathrm{Y}$ & 9,5 & 9,4 & 10 & 10 & 11 & 11 & 10 & 10 & 10 & 9,4 \\
\hline $\mathrm{Zn}$ & 88 & 84 & 77 & 60 & 56 & 55 & 52 & 50 & 51 & 50 \\
\hline
\end{tabular}

As concentrações estão em mg kg-1 quando não especificadas e foram obtidas por ICPAES e ICPMS (apenas Ag, As, Bi, Cd, Ga, Sb, Sc, Ta e Te). O "background" geoquímico litogênico está representado pelos sedimentos da base do testemunho (12 a $31 \mathrm{~cm}$ ). Incerteza de até $15 \%$ é admitida para os dados

cimento foram apresentados pelos elementos com distribuição aproximadamente normal (ou aproximadamente log-normal), esse patamar foi adotado neste trabalho como limitante das incertezas quanto às anomalias. Portanto, acima de três fatores, os enriquecimentos foram tratados como anômalos. Desta forma, o Sc, embora com CV $=4$, apresentou $\mathrm{FE}<3$ e, mediante a margem de incertezas, não foi tratado como elemento anômalo. $\mathrm{O} \mathrm{Cr}, \mathrm{Nb}, \mathrm{Ni}$ e $\mathrm{Y}$, ao contrário, com valores de $\mathrm{CV}$ próximos a 0,3 , mostraram-se localmente anômalos com base no FE, conforme segue abaixo. As incertezas quanto às anomalias são decorrentes dos efeitos litogênicos nos sedimentos (e.g. granulometria, mineralogia, heterogeneidade na fonte), possíveis erros analíticos e de amostragem. A utilização do CV, apenas, para definição de anomalias pode ter um complicador se a anomalia for pontual, diminuta e representada por um pequeno número de amostras, cujas concentrações anômalas serão mascaradas. Assim, o CV é somente um indicativo e as anomalias deverão ser discutidas com base em outros critérios, como é o caso do fator de enriquecimento que revela contrastes geoquímicos pontuais.

A Tabela 3 apresenta um resumo dos elementos geoquimicamente anômalos por setor de amostragem, com indicação dos coeficientes de variação, médias do "background" litogênico, concentrações anômalas e fatores de enriquecimento máximo. Os elementos mais fortemente anômalos (FE máximos entre 49 e 18), em ordem decrescente, foram $\mathrm{Hg}, \mathrm{Mn}, \mathrm{La}, \mathrm{Ca}, \mathrm{Sr}$ e $\mathrm{Cd}$, cujos $\mathrm{CV}$

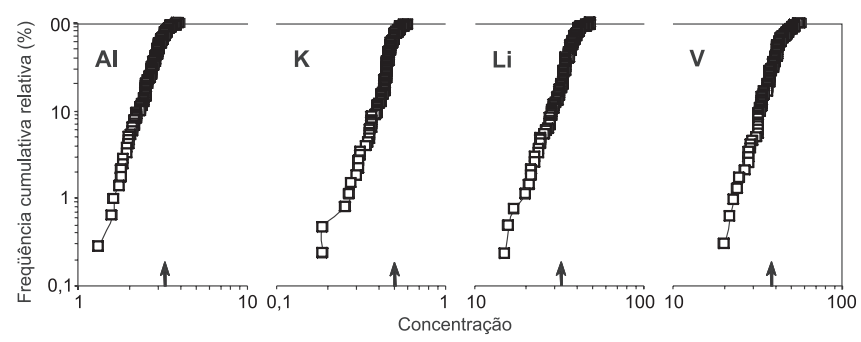

Figura 2. Exemplos de projeções bi-logarítmicas de freqüências cumulativas relativas vs. concentrações de $\mathrm{Al}(\%), \mathrm{K}(\%), \mathrm{Li}\left(\mathrm{mg} \mathrm{kg}^{-1}\right)$ e $\mathrm{V}\left(\mathrm{mg} \mathrm{kg}^{-1}\right)$ nos sedimentos (fração $<63 \mu \mathrm{m}$ ) de superfície e do testemunho CAS-1 da área de estudo. A distribuição aproximadamente log-normal em todos os casos sugere ausência de anomalia ${ }^{29}$. As setas indicam os valores médios do "background" geoquímico litogênico (sedimentos da base do testemunho CAS-1)

oscilaram entre 0,7 e 1,7. Em menor grau (FE máximos entre 16 e 3,1), ocorreram $\mathrm{Zn}, \mathrm{Pb}, \mathrm{Ba}, \mathrm{Cu}, \mathrm{Cr}, \mathrm{Fe}, \mathrm{Nb}, \mathrm{Y}, \mathrm{Ni}$ e $\mathrm{Ga}$, todos com valores de $\mathrm{CV} \leq 0,6$.

A Figura 3 mostra exemplos de elementos cujas concentrações possuem freqüências cumulativas relativas caracteristicamente anômalas, como indicado pela inflexão na parte superior das curvas. Dentre os elementos geoquimicamente anômalos, apenas o $\mathrm{Hg}$ apresentou teores elevados e dispersos $\left(0,1\right.$ a $\left.6,77 \mathrm{mg} \mathrm{kg}^{-1}\right)$ 
por todos os sítios de amostragem investigados. Este aspecto dificultou a observação da assimetria na distribuição das freqüências cumulativas relativas (Figura 3a). Os setores com maior concentração desse metal, em ordem decrescente, foram os rios Cubatão (FE máximo = 49) e Casqueiro (23), o Largo do Caneu (16) e os rios Morrão (6,2), Pedreira $(4,3)$, Cascalho $(4,1)$ e Capivari $(3,1)$ - Tabela 3.

Ao contrário do $\mathrm{Hg}$, as concentrações anômalas de outros metais geralmente ocorreram de forma localizada, especialmente nos sedimentos do rio Morrão (Tabela 3), com destaque para as anomalias de Mn (FE máximo = 48), La (29), Ca (20), Sr (19) e Cd (18). As concentrações de $\mathrm{Mn}$ e La, por ex., chegaram a superar 5000 e 400 $\mathrm{mg} \mathrm{kg}^{-1}$, respectivamente (Figuras 3b-c). Ainda no rio Morrão, outras anomalias foram apresentadas por $\mathrm{Zn}(\mathrm{FE}$ máximo $=16), \mathrm{Pb}$ (12), $\mathrm{Ba}(12), \mathrm{Cu}(11)$ e $\mathrm{Cr}(8,4)$. Neste trabalho, não foi possível quantificar as elevadas concentrações de Fe nos sedimentos do rio Morrão, devido às limitações instrumentais, que mesmo assim projetam um FE superior a 6,8 (concentração > 10\%). Dados inéditos (pesquisa em andamento) estão revelando que a concentração desse metal nos sedimentos do rio Morrão pode ser tão elevada quanto $40 \%$. Anomalias menos expressivas neste rio (FE máximo entre 6,9 e 3,1) foram apresentadas por $\mathrm{Nb}, \mathrm{Y}$, $\mathrm{Ni}$ e $\mathrm{Ga}$.

As concentrações de $\mathrm{Cu}, \mathrm{Ba}$ e $\mathrm{Cd}$, embora se destaquem no rio Morrão, mostraram-se também anômalas em outros rios investiga- dos, com FE máximo oscilando entre 5,1 e 3,8 (Tabela 3). O comportamento do $\mathrm{Cu}$ é destacado na Figura $3 \mathrm{~d}$.

Dentre os elementos-traço com concentrações anômalas identificadas neste trabalho, $\mathrm{Cd}, \mathrm{Cr}, \mathrm{Cu}, \mathrm{Hg}, \mathrm{Mn}, \mathrm{Ni}, \mathrm{Pb}$ e $\mathrm{Zn}$ têm sido reportados como contaminantes do ambiente estuarino estudado, oriundos de várias fontes antrópicas ${ }^{6}$. A origem das anomalias dos outros elementos identificadas neste estudo, no entanto, continua por ser investigada.

Comparando-se os dados deste trabalho, referentes ao sítio de amostragem GD no Largo do Caneu (0,13 $\mathrm{mg} \mathrm{kg}^{-1}$ de Cd; 39 mg kg-1 de Cr; $29 \mathrm{mg} \mathrm{kg}^{-1}$ de Cu; 0,97 mg kg-1 de Hg; 23 mg kg-1 de $\mathrm{Pb} ; 99 \mathrm{mg} \mathrm{kg}^{-1}$ de $\mathrm{Zn}$ ), com aqueles obtidos nos anos de 1979/ $1980^{1}$ (0,19 $\mathrm{mg} \mathrm{kg}^{-1}$ de Cd; $36 \mathrm{mg} \mathrm{kg}^{-1}$ de $\mathrm{Cr} ; 18 \mathrm{mg} \mathrm{kg}^{-1}$ de $\mathrm{Cu}$; $1,16 \mathrm{mg} \mathrm{kg}^{-1} \mathrm{de} \mathrm{Hg} ; 18 \mathrm{mg} \mathrm{kg}^{-1}$ de $\mathrm{Pb} ; 108 \mathrm{mg} \mathrm{kg}^{-1}$ de $\mathrm{Zn}-$ sítio 3 - Figura 1), nota-se que os valores de concentração do passado e os atuais são muito próximos. Portanto, embora não tenha havido uma aparente diminuição da concentração com o tempo (18 anos de intervalo), houve uma relativa estabilidade nos níveis destes metais desde a implantação do programa de controle da contaminação na região, em $1984^{6}$.

Os resultados geoquímicos aqui apresentados sugerem que os rios Cubatão (elevadas concentrações de $\mathrm{Hg}$ ) e Morrão (altas concentrações multi-elementares) são de fato as principais vias de entrada de contaminantes metálicos no sistema estuarino. A conta-

Tabela 3. Resumo da avaliação de elementos geoquimicamente anômalos na fração $<63 \mu \mathrm{m}$ dos sedimentos de superfície da área de estudo (amostragem 1997-1998)

\begin{tabular}{|c|c|c|c|c|c|}
\hline Elementos & $\mathrm{CV}$ & Média do "background" & Anomalias $^{[1]}$ & $\mathrm{FE}$ & Setores \\
\hline \multirow[t]{7}{*}{$\mathrm{Hg}$} & 0,7 & $0,12 \pm 0,04$ & $0,65-6,77$ & 49 & rio Cubatão \\
\hline & & & $0,25-3,04$ & 23 & rio Casqueiro \\
\hline & & & $0,47-1,79$ & 16 & Largo do Caneu \\
\hline & & & $0,37-0,58$ & 6,2 & rio Morrão \\
\hline & & & $0,57-0,61$ & 4,3 & rio Pedreira \\
\hline & & & $0,4-0,57$ & 4,1 & rio Cascalho \\
\hline & & & 0,4 & 3,1 & rio Capivari \\
\hline \multirow[t]{2}{*}{$\mathrm{Mn}$} & 1,7 & $226 \pm 11$ & $3276-5699$ & 48 & rio Morrão \\
\hline & & & $964-1045$ & 4 & rio Cascalho \\
\hline $\mathrm{La}$ & 1,1 & $20 \pm 1$ & $75-450$ & 29 & rio Morrão \\
\hline $\mathrm{Ca}$ & 1,4 & $0,27 \pm 0,05 \%$ & $2,24-4,37 \%$ & 20 & rio Morrão \\
\hline $\mathrm{Sr}$ & 1,3 & $50 \pm 8$ & $214-764$ & 19 & rio Morrão \\
\hline \multirow[t]{2}{*}{$\mathrm{Cd}^{[2]}$} & 0,9 & $0,11 \pm 0,01$ & $0,50-1,6$ & 18 & rio Morrão \\
\hline & & & 0,3 & 3,8 & rio Casqueiro \\
\hline $\mathrm{Zn}$ & 0,5 & $51 \pm 1$ & $406-476$ & 16 & rio Morrão \\
\hline $\mathrm{Pb}$ & 0,6 & $15 \pm 1$ & $92-127$ & 12 & rio Morrão \\
\hline \multirow[t]{3}{*}{$\mathrm{Ba}$} & 0,5 & 30 & $166-196$ & 12 & rio Morrão \\
\hline & & & $98-184$ & 4,9 & rio Cubatão \\
\hline & & & $99-128$ & 3,4 & Largo do Caneu \\
\hline \multirow[t]{4}{*}{$\mathrm{Cu}$} & 0,4 & $14 \pm 1$ & 68-109 & 11 & rio Morrão \\
\hline & & & $48-89$ & 5,1 & rio Cascalho \\
\hline & & & $28-73$ & 4,9 & rio Casqueiro \\
\hline & & & $45-57$ & 3,7 & rio Cubatão \\
\hline $\mathrm{Cr}$ & 0,3 & 31 & $117-138$ & 8,4 & rio Morrão \\
\hline $\mathrm{Fe}^{[3]}$ & $>0,3$ & $2,9 \pm 0,1 \%$ & $>10 \%$ & $>6,8$ & rio Morrão \\
\hline $\mathrm{Nb}$ & 0,3 & $2,1 \pm 0,2$ & $5-11$ & 6,9 & rio Morrão \\
\hline $\mathrm{Y}$ & 0,3 & $10 \pm 0,3$ & $34-48$ & 6 & rio Morrão \\
\hline $\mathrm{Ni}$ & 0,2 & $14 \pm 1$ & $33-39$ & 4,4 & rio Morrão \\
\hline $\mathrm{Ga}$ & 0,4 & $6,3 \pm 0,2$ & 16 & 3,1 & rio Morrão \\
\hline
\end{tabular}

A média do "background" litogênico equivale à geoquímica dos sedimentos da base do testemunho CAS-1 (amostragem 2001). CV = coeficiente de variação (166 amostras) e $\mathrm{FE}=$ fator de enriquecimento (valores máximos). O "background" e as anomalias estão em mg kg-1 quando não especificados. ${ }^{[1]}$ Balizadas pelo $\mathrm{FE}>3,0 \cdot{ }^{[2]}$ Dentre as 166 análises de $\mathrm{Cd}, 119$ valores de concentração foram inferiores ao limite de detecção $\left(0,2 \mathrm{mg} \mathrm{kg}^{-1}\right.$ - ICPAES) e não foram considerados no cálculo do $\mathrm{CV} \cdot{ }^{[3]} \mathrm{A}$ concentração de Fe no rio Morrão foi superior a $10 \%$ (limite máximo de detecção). O valor 10 foi utilizado nos cálculos do CV e FE. 

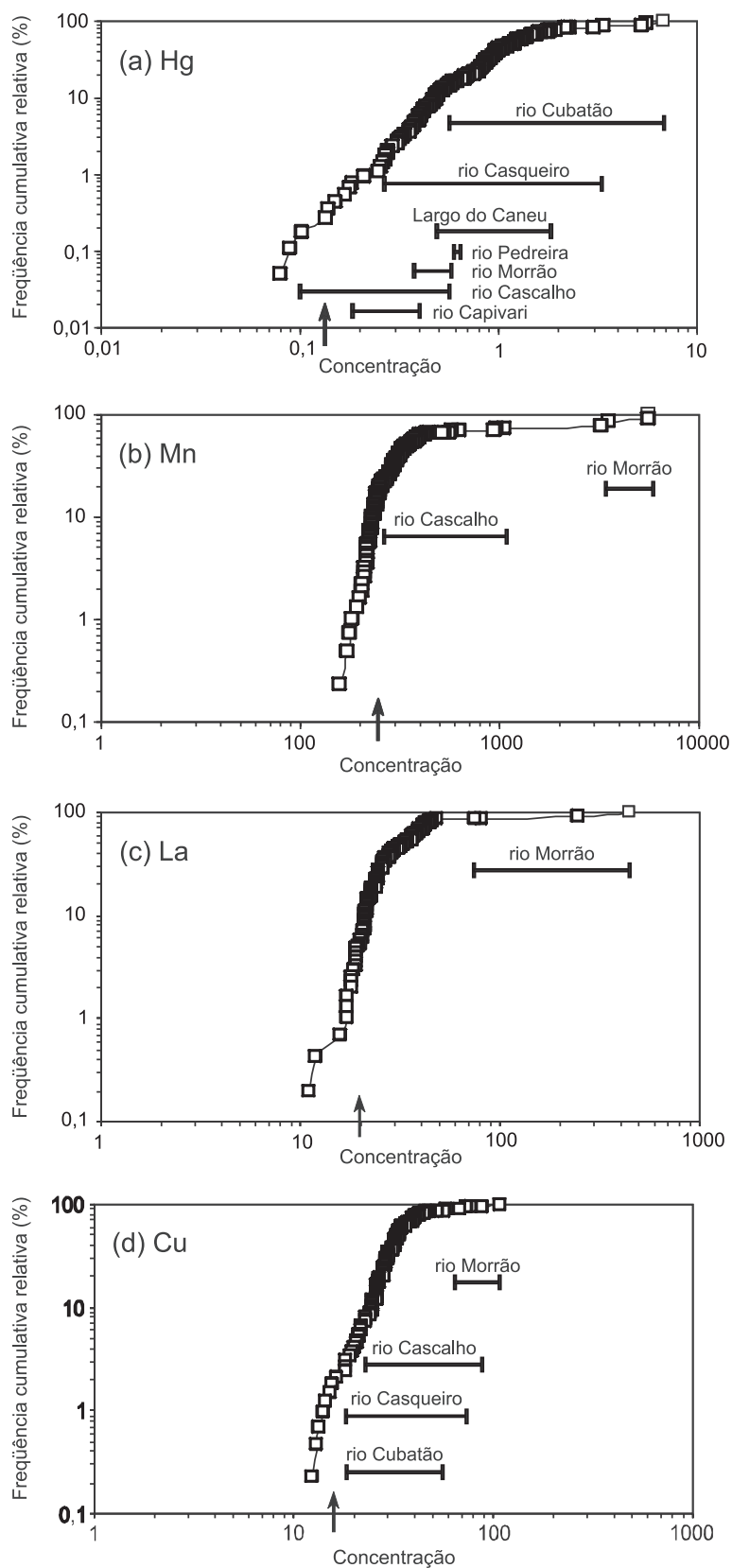

Figura 3. Exemplos de projeções bi-logarítmicas de freqüências cumulativas relativas vs. concentrações $\left(\mathrm{mg} \mathrm{kg}^{-1}\right)$ de (a) $\mathrm{Hg}$, (b) Mn, (c) La e (d) Cu nos sedimentos (fração $<63 \mu \mathrm{m}$ ) de superfície e do testemunho CAS-1 da área de estudo. Um desvio da distribuição log-normal, marcado pela inflexão na parte superior da projeção $(b-d)$, pode ser visto como uma anomalia de acordo com Matschullat et al. ${ }^{29}$. Este desvio não é evidente em (a), em virtude dos valores de concentração de $\mathrm{Hg}$ serem dominantemente bem superiores à média do "background" geoquímico litogênico representada pela seta (concentração média dos sedimentos da base do testemunho CAS-1)

minação, especialmente por $\mathrm{Hg}$, no rio Cubatão é conhecida desde o final da década de $1970^{10}$, mas a importância do rio Morrão como potencial dispersor multi-elementar constitui um novo enfoque de avaliação ambiental na área. Nos sedimentos deste rio, as concentrações de $\mathrm{Cr}, \mathrm{Cu}, \mathrm{Pb}$ e $\mathrm{Zn}$, por ex., enquadram-se na classificação de sedimentos altamente poluídos, de acordo com as normas da Agência de Proteção Ambiental Norte-americana (em mg kg-1, $\mathrm{Cr}$ $>75, \mathrm{Cu}>50, \mathrm{~Pb}>60$ e $\mathrm{Zn}>200)^{36}$.
Tabela 4. Variações sazonais no sistema estuarino de SantosCubatão no ano de 1998, referentes às principais anomalias geoquímicas nos sedimentos (fração $<63 \mu \mathrm{m}$ ) de superfície do rio Morrão

\begin{tabular}{lcccc}
\hline & $\begin{array}{c}\text { Verão } \\
(\text { março) }\end{array}$ & $\begin{array}{c}\text { Outono } \\
\text { (junho) }\end{array}$ & $\begin{array}{c}\text { Inverno } \\
\text { (setembro) }\end{array}$ & $\begin{array}{c}\text { Primavera } \\
\text { (dezembro) }\end{array}$ \\
\hline $\mathrm{Mn}$ & 3276 & 5578 & 5699 & 3551 \\
$\mathrm{La}$ & 450 & 81 & 75 & 248 \\
$\mathrm{Ca}(\%)$ & 4,37 & 2,27 & 2,24 & 3,6 \\
$\mathrm{Sr}$ & 764 & 216 & 214 & 587 \\
$\mathrm{Cd}$ & 1,6 & 0,2 & 0,6 & 0,5 \\
$\mathrm{Zn}$ & 454 & 406 & 424 & 476 \\
$\mathrm{~Pb}$ & 127 & 92 & 96 & 101 \\
$\mathrm{Ba}$ & 195 & 187 & 166 & 196 \\
$\mathrm{Cu}$ & 108 & 68 & 78 & 109 \\
$\mathrm{Cr}$ & 117 & 134 & 138 & 120 \\
$\mathrm{Nb}$ & 10 & 6 & 5 & 11 \\
$\mathrm{Hg}$ & 0,58 & 0,37 & 0,41 & 0,42 \\
$\mathrm{Y}$ & 48 & 14 & 14 & 34 \\
$\mathrm{Ni}$ & 39 & 33 & 34 & 38 \\
\hline $\mathrm{As}$ & & & 34 & \\
\hline
\end{tabular}

As concentrações estão em $\mathrm{mg} \mathrm{kg}^{-1}$ quando não especificadas. Incerteza de até $15 \%$ é admitida para os dados. Os elementos estão em ordem decrescente de fatores de enriquecimento no rio Morrão

\section{Variabilidade sazonal}

Uma variação sazonal substancial das concentrações dos elementos anômalos foi freqüentemente observada. Dentre as drenagens investigadas, o rio Morrão foi o que apresentou variabilidade geoquímica sazonal mais sistemática, a qual é mostrada na Tabela 4. Os resultados sugerem maior concentração da maioria dos elementos analisados, incluindo, por ex., $\mathrm{Cu}, \mathrm{Cd}, \mathrm{Hg}$ e $\mathrm{Pb}$, no verão e/ou na primavera. Ao contrário destes elementos, o Mn exibiu maiores concentrações durante o outono e o inverno. A variabilidade sazonal do Fe não pôde ser avaliada em virtude de sua concentração superar o limite máximo analítico de $10 \%$.

Muitos estudos têm avaliado os mecanismos envolvidos na variabilidade geoquímica temporal de sedimentos costeiros, sendo relatado que as condições físico-químicas das águas superficiais podem regular a precipitação ou solubilização dos metais ${ }^{9,37}$. Sholkovitz e Szymczak ${ }^{38}$, estudando a foz do Rio Amazonas, mostraram que o aumento da salinidade proporcionou incremento na adsorção de elementos terras raras nos sedimentos. Por outro lado, Lau ${ }^{39}$, em um estuário subtropical, admitiu que incrementos na salinidade e temperatura promoveram maior solubilidade de metais, diminuindo sua concentração nos sedimentos. Existe maior consenso sobre a influência de outros fatores na fixação ou mobilização de metais em sedimentos costeiros, como as condições redox e de acidez, que determinarão a estabilidade dos compostos envolvidos na retenção dos contaminantes ${ }^{8,40}$. Estes fatores podem ser significativamente influenciados por variações sazonais na temperatura e na pluviosidade ${ }^{13,41}$. Não obstante, as oscilações temporais no aporte antrópico de elementos-traço também podem contribuir para a variabilidade sazonal das concentrações destes contaminantes nos sedimentos costeiros, como no caso da área de influência da região metropolitana de Fortaleza, no Ceará ${ }^{25}$.

Considerando todo o conjunto de drenagens investigado (dados não mostrados), alguns dos contaminantes tenderam a seguir a mesma variabilidade sazonal observada no rio Morrão (e.g. Cd $\mathrm{e} \mathrm{Pb}$ ), enquanto outros não mostraram respostas sazonais consistentes (por ex., $\mathrm{Cu}, \mathrm{Hg}$ e $\mathrm{Mn}$ ). Esta situação pode ser atribuída, 
por ex., à influência de fontes difusas de contaminação e/ou ao transporte dos sedimentos entre os períodos de amostragem, como parece ser refletido pela variação temporal na granulometria das amostras deste estudo. Este transporte implica na movimentação de plumas de contaminação das diferentes drenagens e possivelmente influencia as concentrações dos elementos nos sedimentos. No rio Morrão, a consistência dos dados geoquímicos indica que esse processo não afetou os resultados, possivelmente devido à fonte principal de contaminação estar próxima ao sítio de amostragem - uma grande siderúrgica instalada a $\sim 1,5 \mathrm{~km}$ à montante do sítio $\mathrm{GJ}^{6}$.

Os elevados níveis dos elementos-traço no sistema estuarino podem gerar riscos de contaminação da biota. Investigações detalhadas, levando em conta as variações sazonais, devem ser conduzidas para se avaliar o potencial impacto dessa contaminação na fauna e flora da região. Embora estudos realizados pela CETESB em $1999^{6}$ tenham revelado baixas concentrações de $\mathrm{Cd}, \mathrm{Cr}, \mathrm{Cu}, \mathrm{Hg}, \mathrm{Mn}, \mathrm{Ni}, \mathrm{Pb}$ e $\mathrm{Zn}$ na água do estuário e em alguns organismos (peixes, siris, mexilhões, ostras e caranguejos), é importante destacar que não foram consideradas amostragens sazonais e o rio Morrão não foi incluído na investigação. A falta de avaliações sazonais da contaminação de organismos por estudos já realizados ${ }^{1,6}$ e os resultados aqui apresentados demonstram a importância de se investigar a variação temporal da contaminação da biota na área investigada. Particularmente importante é o risco de biomagnificação dos contaminantes ao longo da cadeia trófica, que pode atingir espécies de aves migratórias e ameaçadas de extinção.

\section{CONCLUSÕES}

O presente estudo mostra que os sedimentos do sistema estuarino de Santos-Cubatão alcançaram níveis de concentração multi-elementar que podem afetar o equilíbrio do ecossistema local. $\mathrm{O} \mathrm{Hg}$ apresentou a maior dispersão nos sedimentos do estuário, influenciada provavelmente por origens difusas, apresentando concentrações mais significativas no rio Cubatão. Os demais elementos apresentaram maiores concentrações, especialmente nos sedimentos do rio Morrão. Uma variabilidade geoquímica sazonal foi freqüentemente encontrada, em especial nos sedimentos do rio Morrão. Nas demais drenagens monitoradas, a variação sazonal das concentrações de alguns elementos não foi conclusiva, possivelmente devido à influência de fontes difusas de contaminação. Porém, foi evidenciada a importância de análises sazonais para futuros monitoramentos da qualidade dos sedimentos do sistema estuarino estudado. Metais geoquimicamente anômalos, como $\mathrm{Cd}, \mathrm{Cr}, \mathrm{Cu}$, $\mathrm{Hg}, \mathrm{Mn}, \mathrm{Pb}$ e $\mathrm{Zn}$, assim como elementos terras raras (aqui representados pelo La) são altamente recomendáveis para investigações futuras quanto ao risco à biota, em especial às aves residentes e migratórias, diretamente expostas ao contato com os sedimentos e à ingestão de organismos bentônicos.

\section{AGRADECIMENTOS}

Ao CNPq (R. H. R. Matos), à CAPES (G. C. Kristosch), ao FAEPEX-UNICAMP (W. Luiz-Silva, processo № 676/03) e à FAPESP (W. Luiz-Silva, processo № 04/00059-6) pelo apoio financeiro que permitiu a execução dessa pesquisa; à UNESP-Rio Claro (IB e IGCE) e à UNICAMP (IG) pela utilização de laboratórios; ao Prof. Dr. R. S. Angélica e a dois revisores anônimos pelas críticas e sugestões sobre o manuscrito original.

\section{REFERÊNCIAS}

1. Boldrini, V. B.; Navas-Pereira, D.; Ambiente 1987, 1, 118.

2. Gutberlet, J.; Cubatão: desenvolvimento, exclusão social e degradação ambiental, Edusp: São Paulo, 1996.

3. Braga, E. S.; Bonetti, C. V. D. H.; Burone, L.; Bonetti Filho, J.; Mar. Pollut. Bull. 2000, 40, 165 .

4. Luiz-Silva, W.; Matos, R. H. R.; Kristosch, G. C.; Quim. Nova 2002, 25, 753.

5. Adams, P.; Odious debts: loose lending, corruption and the third world's environmental Legacy, Earthscan: Toronto, 1991.

6. Companhia de Tecnologia e Saneamento Ambiental; $O$ sistema estuarino de Santos e São Vicente, CETESB: São Paulo, 2001.

7. Santos Filho, E.; Silva, R. S.; Barretto, H. H. C.; Inomata, O. N. K.; Lemes, V. R. R.; Sakuma, A. M.; Scorsafava, M. A.; Rev. Saúde Pública 1993, 27 , 59.

8. Harbison, P.; Mar. Pollut. Bull. 1986, 17, 246.

9. Warnken, K. W.; Gill, G. A.; Griffin, L. L.; Santschi, P. H.; Mar. Chem. 2001, 73, 215.

10. Companhia de Tecnologia e Saneamento Ambiental; Metais pesados na Baía de Santos e estuários de Santos e São Vicente, CETESB: São Paulo, 1981.

11. Lau, S. S. S.; Chu, L. M.; Water Res. 1999, 34, 379.

12. Aragon, G. T.; Ovalle, A. R. C.; Carmouze, J. P.; Mangroves and Salt Marshes 1999, 3, 85.

13. Gueiros, B. B.; Machado, W.; Lisboa Filho, S. D.; Lacerda, L. D.; J. Coastal Res. 2003, 19, 550

14. Almeida, F. F. M.; Carneiro, C. D. R.; Rev. Bras. Geociências 1998, 28, 135

15. Matos, R. H. R.; Tese de Doutorado, Universidade Estadual Paulista, Brasil, 2002.

16. Kristosch, G. C.; Tese de Doutorado, Universidade Estadual Paulista, Brasil, 2003.

17. Rebello, A. L.; Haekel, W.; Moreira, I.; Santelli, R.; Schroeder, F.; Mar. Chem. 1986, 18, 215.

18. Lacerda L. D.; Carvalho, C. E. V.; Rezende, C. E.; Pfeiffer, W. C.; Mar. Pollut. Bull. 1993, 26, 220.

19. Silva, L. F. F.; Machado, W.; Lisboa Filho, S. D.; Lacerda, L. D.; Water, Air, Soil Pollut. 2003, 145, 67.

20. Soares, H. M. V. M.; Boaventura, R. A. R.; Machado, A. A. S. C.; Esteves da Silva, J. C. G.; Environ. Pollut. 1999, 105, 311.

21. Villaescusa-Celaya, J. A.; Gutiérrez-Galindo, E. A.; Flores-Munõz, G.; Environ. Pollut. 2000, 108, 453.

22. U.S. Environmental Protection Agency; Methods for collection, storage and manipulation of sediments for chemical and toxicological analyses: technical manual, U.S. EPA: Washington, 2001.

23. Frignani, M.; Bellucci, L. G.; Langone, L.; Muntau, H.; Mar. Chem. 1997, $58,275$.

24. Machado, W.; Moscatelli, M.; Rezende, L. G.; Lacerda, L. D.; Environ. Pollut. 2002, 120, 455.

25. Marins, R. V.; Paula Filho, F. J.; Maia, S. R. R.; Lacerda, L. D.; Marques, W. S.; Quim. Nova 2004, 27, 763.

26. Licht, O. A. B.; Prospecção geoquímica: princípios, técnicas e métodos, CPRM: Rio de Janeiro, 1998.

27. Koch Jr., G. S.; Link, R. F.; Statistical analysis of geological data, Willey: New York, 1971.

28. Lepeltier, C.; Econ. Geol. 1969, 64, 538.

29. Matschullat, J.; Ottenstein, R.; Reimann, C.; Environ. Geol. 2000, 39, 990.

30. Gresens, R. L.; Chem. Geol. 1967, 2, 47.

31. Loring, D. H.; Mar. Chem. 1990, 29, 155.

32. Hoshika, A.; Suguio, K.; Hirata, S.; Tessler, M. G.; Mishima, Y.; Mahiques, M. M.; Actas do $3^{\circ}$ Simpósio de Oceanografia, São Paulo, Brasil, 1996.

33. Turekian, K. K.; Wedepohl, K. H.; Geol. Soc. Amer. Bull. 1961, 72, 175.

34. Sadowski, G. R.; Tese de Doutorado, Universidade de São Paulo, Brasil, 1974.

35. Instituto Geográfico e Geológico de São Paulo; Carta topográfica de Santos. Folhas SF-23-Y-D-IV-3 e SG-23-V-B-I-1. Escala 1 / 50.000, 1 a ed., IGG: São Paulo, 1972.

36. Nichols, S. J.; Manny, B. A.; Schloesser, D. W.; Edsall, T. A.; Hydrobiologia 1991, 219, 307.

37. Cooper, D. C.; Morse, J. W.; Environ. Sci. Technol. 1998, 32, 327.

38. Sholkovitz, E.; Szymczak, R.; Earth Planet. Sci. Lett. 2000, 179, 299.

39. Lau, S. S. S.; Water Res. 2000, 34, 387.

40. Calmano, W.; Hong, J.; Förstner, U.; Water Sci. Technol. 1993, 28, 223

41. Otero, X. L.; Macias F.; Biogeochem. 2002, 61, 247. 